\title{
Prevalence and Measurement of Anxiety and Depression in Working Nurses in Nepal: A Reflection of Professional Hazard in Low Income Nation
}

\section{Pramila Karki}

Vatsalya IVF Center

George W. B. J. Katwal

Annapurna Neurological Institute and Allied Sciences

Ayush Chandra

Tianjin Medical University General Hospital

Avinash Chandra ( $\sim$ chandraavi@gmail.com)

Annapurna Neurological Institute and Allied Sciences https://orcid.org/0000-0002-3895-5369

Research article

Keywords: anxiety, depression, nurses, nepal

Posted Date: November 6th, 2019

DOl: https://doi.org/10.21203/rs.2.16888/v1

License: (c) (1) This work is licensed under a Creative Commons Attribution 4.0 International License.

Read Full License 


\section{Abstract}

Background Anxiety, Depression is one of the under reported, underdiagnosed in Nepal though it is the leading cause of incapacitation and is the leading healthcare burden worldwide. The prevalence of anxiety, depression among health personnel is not reported. In this study we attempted to assess the incidence and impact of depression and anxiety in nurses who are working upfront in different hospitals. Method A cross-sectional study was carried out and the sample was collected from nurses working in different hospitals who had spent 6 months or more time as a working nurse in those hospitals. Prevalence of anxiety and depression was assessed using a structured validated questionnaire, The Hamilton Anxiety Scale (HAM-A) with a cut-off score for various levels of anxiety. Similarly, General Anxiety Disorder Questionnaires (GAD), Hamilton Depression Rating Scale (HAM-D) was used to assess depression and anxiety.Result The analysis of these different scales revealed that disabling anxiety prevailed at highest (43.6\%) in nursing staff according to HAM-A scale. Moderate anxiety also seemed to be higher $(>20 \%)$ in GAD questionnaire. The moderate to severe anxiety prevailed in majority of our study participants in all these scales (HAM-A, HAM-D, GAD). Interestingly, there seemed a strong association between HAM-A to GAD ( $p=0.008)$.Conclusions This is probably the first study of its kind that reports on the prevalence of anxiety, depression in the nurses who work in different hospitals in Nepal. We hope this study also depicts the mental health problems that the medical professionals are suffering in other nations as well.

\section{Background}

Anxiety and depression are one of the most common mental health issues. Several research reports on the prevalence of depression and anxiety on medical, nursing students. [1] The high intensity of training, large targets to achieve are some of the important reasons for the depression and anxiety in them. Systematic reviews and meta-analyses indicated that the prevalence of anxiety and depression among medical students worldwide was around $28 \%$, and suicidal ideation was $11.1 \%$.[2-4] However, not many papers report the prevalence of anxiety and depression among nurses employed in hospitals. [5] However each papers from around the world on depression symptoms indicate the prevalence of anxiety symptom from $18 \%$ to $40 \%$ in nurses in United States, $11 \%$ to $80 \%$ in Iranian nurses,[6] 35\% in Chinese nurses[7] while $41.2 \%$ in Australian nurses. [8] A survey done in Vietnam showed that the prevalence of depression and anxiety is higher than the prevalence of anxiety and depression in general population of that country. $[9,10]$ The low socio-economic status, longer working period, uncertainty for the future and low earning than the counterparts working in abroad countries add the fuel to the fire of anxiety and depression.

In this study we have attempted to see the prevalence of anxiety and depression among nurses who are working in different hospitals in Nepal. Depression in healthcare workers should be addressed as it may significantly impact the quality of care they provide to the consumers. Nurses regularly face a various types of stressors including but not limited to : work hours, meeting patient's needs, and the patient attendant's expectations, lack of professional support.[8] The greatest concern is that the depressed staff often exhibit low mood, difficulty focusing and as a result are accident prone and lower total work output 
and decreased efficiency. $[11,12]$ The accurate measurement and identification of depression and anxiety is challenging because of frequent overlapping or co-occurrence of these conditions.

\section{Methods}

This was an observational cross-control study to examine the prevalence of anxiety and depression in a cohort of Nepalese nurses working in different hospitals. Data were collected from 64 clinically active nurses working in different hospitals in Nepal. The data were collected in 2 months duration (July-August 2019). Prior to inclusion, all participants were screened for chronic diseases including previously diagnosed clinical depression, or other mental health issues and were excluded from the study if found. The personal history for the exclusion was chronic smoking habits (>10 cigarettes per day), alcohol intake (more than 16 standard drinks per day). Data were collected using the different set of questionnaires (HAM-A, HAM-D and GAD) were used for the assessment of anxiety and depression.

The level of anxiety depression was based on the scores and their respective cutoff points. HAM-A consisted of mild, moderate, severe, grossly disabling depression, while GAD included as mild, moderate, and severe based on their scores respectively. HAM-A is a checklist of self-answerable questionnaires that assist a clinician in evaluating each participant as to the degree of anxiety and pathological condition. It is one of the first rating scales developed to measure the severity of anxiety symptoms and is still widely used today in both clinical and research settings. It consists of 14 items -, each defined by a series of symptoms. The total score range of 0-56, where less than 17 indicated mild severity, 18-24 mild to moderate severity and 25-30 as moderate to severe. [13] GAD is also another easy to perform initial screening tool for generalized anxiety disorder. We have used 7 elements of GAD questionnaires, also called as GAD7 and it is obtained by adding score for each question. The result was grouped according to the score achieved. From score 0-5 was grouped as mild anxiety, 6-10 as moderate anxiety, 11-15 as severe anxiety. Using a threshold of 10 , it has shown fair amount of Sensitivity ( $>89 \%)$ and Specificity $(>82 \%)$. [14] Data were entered and analyzed with SPSS (version 20). The descriptive statistical analysis was run through the data. The frequency was calculated using descriptive analysis and the association between different scales (HAM-A and GAD) and (HAM-D and GAD) was analyzed by using analysis of variance (ANOVA) one way.

\section{Results}

Interestingly, only female pursue the career as a nurse in Nepal and hence our study consisted of only female sex. Unfortunately, we could not show the gender difference in anxiety and depression in our study because of the above reason. The analysis for each of the scales used in this study was done. The analysis revealed that highest number of participants (43.6\%) were suffering from disabling anxiety according to HAM-A scale. Apart from that, $20.5 \%$ of participants had mild and $9 \%$ had severe anxiety. None of our participants denied any anxiety. In GAD scoring, the analysis revealed that $25.6 \%$ of our participants suffered from moderate anxiety, while $10.8 \%$ had severe anxiety and $16.7 \%$ had mild anxiety. Like the HAM-A scale, none of our GAD participants denied anxiety. There was a strong correlation 
between HAM-A and GAD as well as in HAM-D and GAD in our participants which was seen in ANOVA. The association between HAM-D and GAD had significant positive correlation $(p=0.016)$, and the association between HAM-A and GAD even showed stronger positive correlation $(p=0.008)$.

\section{Discussion}

There is a vast body of literature on anxiety and depression on general population but not much on working nurses and very few on low economic countries. Anxiety, Depression was measured using HAMD, HAM-A, and GAD 7 scales. HAM-D consisted of 21 questions among which if the participants are diagnosed as from none to severe anxiety according to the score, they get up to the $17^{\text {th }}$ questions. HAMA consists of 14 sets of questions and the score of $<17$ denotes mild severity while $>25$ denotes severe. GAD 7 consists of 7 sets of questions and $<5$ is mild while $>10$ is severe. Nepal falls in a low and middleincome countries and the social anxiety disorder is much prevalent in these nations[15], yet only few literatures emphasize on the mental health issues in health personnel especially on the nurses who are not the students anymore. In nurses who are working, it is expected to have comparatively lesser anxiety in the employed groups rather than the student group as they have no more study or exams or other procedures to perform. Contrary to this, our study revealed that the anxiety level is very prevalent in the employed nurses. It aligns with the analysis done by Letvak et al, and Mahraj et al, in which they found that the hospital employed nurses have higher rates of depressive and anxiety symptoms.[5, 8] Similar kind of result in our study could be mainly due to the poor working condition, low salary jobs and as a result low satisfaction to the job. On addition to these, highly stressful job, and low number of staff also could be the reason for anxiety and depression. The low job satisfaction is one of the reasons for the anxiety symptoms among working nurses as found in a study on German nurses.[16] There can be serious consequences to the situation where staff work under extreme anxiety and stress. Several researches have shown that poor mental health may led to decrease in cognitive performances and ultimately resulting in inadequate performance.[17] Our study points out several important aspects of mental health in working nurses that needs to be considered. There are some limitations (such as quite small representative sample and cross-sectional observational study design) in our study, and hence further research seems inevitable. Further research may benefit from larger sample, and longitudinal type of study as well as more elements to be included. A more comprehensive assessment of demographic, personal and work-related factors need to be included as well.

\section{Conclusions}

Nursing is an indispensable part of our health care system. Patient care, hospitalization rely heavily upon their ability to work optimally and without them it would be impossible to deliver the best possible care. Thus, the increasing anxiety depression among nurses cannot be afforded to ignore. The higher prevalence of anxiety depression among nurses as shown through our study is a witness of the fact that the working situation of nurses need to be considered in due time. The anxiety among working nurses 
may not be totally preventable but the recognition of the problem would certainly bring many measures to curb it down.

The limitation of this study was that it consisted of a smaller sample, with homogenous gender. Thus, further bigger research is needed to help care for the well-being of nurses and minimize poor mental health in workplace. This study certainly calls for the policy makers to consider the situation and bring out any short term and long-term support strategies and interventions that would combat the psychological stress and exhaustion and thus improve the mental health of working nurses.

\section{Abbreviations}

HAM-A: The Hamilton Anxiety Scale

GAD: General Anxiety Disorder Questionnaires

HAM-D: Hamilton Depression Rating Scale

\section{Declarations}

Ethics approval and consent to participate

This study was reviewed and approved by the institutional review committee (IRC) of Annapurna Neurological Institute and Allied Sciences. It was conducted in accordance with the Declaration of Helsinki. Additionally, all participants who agreed to participate were only enrolled after giving written informed consent.

Acknowledgement

None

Funding

The authors received no financial support for the research, authorship or whatsoever related to the publication.

Author Information

Affiliations

1. Vatsalya IVF Center, Nepal

Pramila Karki

2. of Neurology, Annapurna Neurological Institute and Allied Sciences, Nepal Avinash Chandra, George WBJ Katwal

3. of General Medicine, Tianjing Medical University, China 
Ayush Chandra

Contributions

All authors have made substantial contributions to this manuscript. PK contributed to the conception and drafted the article. GJWK and AC critically revised the article. AC contributed to the statistical analysis, study design

Corresponding Author:

Avinash Chandra, chandraavi@gmail.com

Consent for publication

Not applicable

Competing Interests

None to declare

\section{References}

1. Moir, F., et al., Depression in medical students: current insights. Adv Med Educ Pract, 2018. 9: p. 323333.

2. Puthran, R., et al., Prevalence of depression amongst medical students: a meta-analysis. Med Educ, 2016. 50(4): p. 456-68.

3. Rotenstein, L.S., et al., Prevalence of Depression, Depressive Symptoms, and Suicidal Ideation Among Medical Students: A Systematic Review and Meta-Analysis. JAMA, 2016. 316(21): p. 2214-2236.

4. Tam, W., K. Lo, and J. Pacheco, Prevalence of depressive symptoms among medical students: overview of systematic reviews. Med Educ, 2019. 53(4): p. 345-354.

5. Letvak, S., C.J. Ruhm, and T. McCoy, Depression in hospital-employed nurses. Clin Nurse Spec, 2012. 26(3): p. 177-82.

6. Ardekani, Z.Z., et al., Prevalence of mental disorders among shift work hospital nurses in Shiraz, Iran. Pak J Biol Sci, 2008. 11(12): p. 1605-9.

7. Cheung, T. and P.S. Yip, Depression, Anxiety and Symptoms of Stress among Hong Kong Nurses: A Cross-sectional Study. Int J Environ Res Public Health, 2015. 12(9): p. 11072-100.

8. Maharaj, S., T. Lees, and S. Lal, Prevalence and Risk Factors of Depression, Anxiety, and Stress in a Cohort of Australian Nurses. Int J Environ Res Public Health, 2018. 16(1).

9. Nguyen, D.T., et al., Depression, anxiety, and suicidal ideation among Vietnamese secondary school students and proposed solutions: a cross-sectional study. BMC Public Health, 2013. 13: p. 1195. 
10. Vuong, D.A., et al., Mental health in Vietnam: Burden of disease and availability of services. Asian J Psychiatr, 2011. 4(1): p. 65-70.

11. Schreiber, M.A., J. Differding, and T.J. Esposito, Research: questions and answers from academic trauma surgeons. J Trauma, 2008. 64(4): p. 1113-7; discussion 1117-8.

12. Wang, J., et al., Workplace characteristics, depression, and health-related presenteeism in a general population sample. J Occup Environ Med, 2010. 52(8): p. 836-42.

13. Matza, L.S., et al., Identifying HAM-A cutoffs for mild, moderate, and severe generalized anxiety disorder. Int J Methods Psychiatr Res, 2010. 19(4): p. 223-32.

14. Plummer, F., et al., Screening for anxiety disorders with the GAD-7 and GAD-2: a systematic review and diagnostic metaanalysis. Gen Hosp Psychiatry, 2016. 39: p. 24-31.

15. Stein, D.J., et al., The cross-national epidemiology of social anxiety disorder: Data from the World Mental Health Survey Initiative. BMC Med, 2017. 15(1): p. 143.

16. Schulz, M., et al., Work-related behaviour and experience pattern in nurses: impact on physical and mental health. J Psychiatr Ment Health Nurs, 2011. 18(5): p. 411-7.

17. Marazziti, D., et al., Cognitive impairment in major depression. Eur J Pharmacol, 2010. 626(1): p. 836.

\section{Supplementary Files}

This is a list of supplementary files associated with this preprint. Click to download.

- STROBEchecklist.doc 